\title{
Unsupervised Artificial Neural Networks For Clustering Of Document Collections
}

\author{
Abdel-Badeeh M. Salem, Mostafa M. Syiam, and Ayad F. Ayad \\ Computer Science Department, Faculty of Computer \& Information Sciences \\ Ain Shams University, Cairo, Egypt. \\ Tel. $(+202)$ 6844284, Fax. $(+202) 6828298$
}

\begin{abstract}
The Self-Organizing Map (SOM) has shown to be a stable neural network model for high- dimensional data analysis. However, its applicability is limited by the fact that some knowledge about the data is required to define the size of the network. In this paper the Growing Hierarchical SOM (GHSOM) is proposed. This dynamically growing architecture evolves into a hierarchical structure of self-organizing maps according to the characteristics of input data. Furthermore, each map is expanded until it represents the corresponding subset of the data at specific level. We demonstrate the benefits of this novel model using a real world example from the document-clustering domain. Comparison between both models (SOM \& GHSOM) was held to explain the difference and investigate the benefits of using GHSOM.
\end{abstract}

Key-Words: - Neural networks, Self-Organizing Map, Document Clustering.

\section{Introduction}

The Self-Organizing Map (SOM) [1] is an artificial neural network model that is well suited for mapping high-dimensional data into a 2-dimensional representation space. The training process is based on weight vector adaptation with respect to the input vectors. The SOM has shown to be a highly effective tool for data visualization in a broad spectrum of application domains [2] . Especially the utilization of the SOM for information retrieval purposes in large free-form document collections has gained wide interest in the last few years $[3,4,5]$. The general idea is to display the contents of a document library by representing similar documents in similar regions of the map. One of the disadvantages of the SOM in such an application area is its fixed size in terms of the number of units and their particular arrangement, which has to be defined prior to the start of the training process. Without knowledge of the type and the organization of the documents it is difficult to get satisfying results without multiple training runs using different parameter settings, which obviously is extremely time consuming given the high-dimensional data representation. Recently a number of neural network models inspired by the training process of the SOM and having adaptive architectures were proposed [6]. The model being closest to the SOM is the so-called Growing Grid [7], where a SOM-like neural network grows dynamically during training. The basic idea is to add rows or columns to the SOM in those areas where the input vectors are not yet represented sufficiently. More precisely, units are added to those regions of the map where large deviations between the input vectors and the weight vector of the unit representing these input data are observed. However, this method will produce very large maps, which are difficult to survey and therefore are not that suitable for large document collections. Another possibility is to use a hierarchical structure of independent SOMs [8], where for every unit of a map a SOM is added to the next layer. This means that on the first layer of the Hierarchical Feature Map (HFM) we obtain a rather rough representation of the input space but with descending the hierarchy the granularity increases. We believe that such an approach is especially well suited for the representation of the contents of a document collection. The reason is that document collections are inherently structured hierarchically with respect to different subject matters. This is essentially the way how conventional libraries are organized for centuries. However, like with the original SOM, the HFM uses a fixed architecture with a specified depth of the hierarchy and predefined size of the various SOMs on each layer. Again, we need profound knowledge of the data in order to define a suitable architecture. In order to combine the benefits of the neural network models described above we introduce a Growing Hierarchical SOM (GHSOM). This model consists of a hierarchical architecture where each layer is composed of independent SOMs that adjust their size according to the requirements of the input data. The remainder of the paper is organized as follows. In section 2 we describe the architecture and the training process of the GHSOM. The used data set and preprocessing steps are demonstrated in section 3 . The results of experiments in document clustering with both SOM and GHSOM are provided in section 4. Finally, we present some conclusions in section 5 .

\section{Growing Hierarchical SOM (GHSOM)}

The key idea of the Growing Hierarchical SelfOrganizing Map (GHSOM) is to use a hierarchical 
neural network structure composed of a number of individual layers each of which consists of independent self-organizing maps. In particular, the neural network architecture starts with a single unit $\mathrm{SOM}$ at layer 0 . One SOM is used at layer 1 of the hierarchy. For every unit in this layer 1 map, a SOM might be added to the next layer of the hierarchy. This principal is repeated with the third and any further layers of the GHSOM.

Since one of the shortcomings of the SOM usage is its fixed network architecture in terms of the number units and their arrangement, we rather rely on an incrementally version of the SOM. This relieves us from the burden of predefining the network's size which is now determined during the unsupervised training process according to the peculiarities of the input data space. Pragmatically speaking, the GHSOM is intended to uncover the hierarchical relationship between input data in a straightforward fashion. More precisely, the similarities of the input data are shown in increasingly finer levels of detail along the hierarchy defined by the neural network architecture. SOMs at higher layers give a coarsegrained picture of the input data space whereas SOMs of deeper layers provide fine-grained input discrimination. The growth process of the neural network is guided by the so-called quantization error, which is a measure of the quality of the input data representation.

The starting point for the growth process is the overall deviation of the input data as measured with the single unit SOM at layer 0. This unit is assigned a weight vector $m_{0}, m_{0}=\left[\begin{array}{lll}\mu_{01}, \mu_{02}, \ldots, & \mu_{0 n}\end{array}\right]^{\mathrm{T}}$, computed as the average of all input data. The deviation of the input data, i.e. the mean quantization error of this single unit, is computed as given in expression (1) with $d$ representing the number of input data $x$. The mean quantization error of a unit will be referred to as mqe in lower case letters.

$$
m q e_{0}=\frac{1}{d}\left\|m_{0}-x\right\|
$$

After the computation of $m q e_{0}$, training of the GHSOM starts with its first layer SOM. This first layer map initially consists of a rather small number of units, e.g. a grid of $2 \times 2$ units. Each of theses units $i$ is assigned an $n$-dimensional weight vector $m_{i}, m_{i}=\left[\mu_{i 1}, \mu_{i 2}, \ldots, \mu_{i n}\right]^{\mathrm{T}}, m_{i \in R}{ }^{n}$, which is initialized with random values. It's important to note that weight vectors have the same dimensionality as the input patterns.

The learning process of SOMs may be described as a competition among the units to represent the input patterns. The unit with the weight vector being closest to the presented input pattern in terms of input space wins the competition. The weight vector of the winner as well as units in the vicinity of the winner are adapted in such a way as to resemble more closely the input pattern [9]. The degree of the adaptation is guided by means of a learning rate parameter $\alpha$, decreasing in time. The number of units that are subject to adaptation also decreases in time such that at the beginning of the learning process a large number of units around the winner are adapted, whereas towards the end only the winner is adapted. These units are chosen by means of a neighborhood function $h_{c i}$, which is based on the units' distances to the winner as measured in the 2-dimensional grid formed by the neural network. In combining these principles of SOM training, the learning rule may be written as given in expression (2), where $x$ represents the current input pattern, and $c$ refers to the winner at iteration $t$

$$
m_{i}(t+1)=m_{i}(t)+\alpha(\mathrm{t}) h_{c i}(t)\left[x(t)-m_{i}(t)\right]
$$

In order to adapt the size of this first layer SOM, the mean quantization error of the map is computed ever after a fixed number $\lambda$ of training iterations as given in expression (3). In this formula, $u$ refers to the number of units $i$ contained in the SOM $m$. In analogy to expression (1), $\mathbf{m q}_{i}$ is computed as the average distance between weight vector $m_{i}$ and the input patterns mapped onto unit $i$. The mean quantization error of a map will be referred to as MQE in upper case letters.

$$
M Q E_{m}=\frac{1}{u} \sum_{i} m q e_{i}
$$

The basic idea is that each layer of the GHSOM is responsible for explaining some portion of the deviation of the input data as present in its preceding layer. This is done by adding units to the SOMs on each layer until a suitable size of the map is reached. More precisely, the SOMs on each layer are allowed to grow until the deviation present in the unit of its preceding layer is reduced to at least a fixed percentage $\tau_{m}$. Obviously, the smaller the parameter $\tau_{m}$ is chosen the larger will be the size of the emerging SOM. Thus, as long as $\mathbf{M Q E} \mathbf{E}_{m}>=\tau_{m} \mathbf{m q e}_{0}$ holds true for the first layer map $m$, either a new row or a new column of units is added to this SOM. This insertion is performed neighboring the unit $e$ with the highest mean quantization error, mqe $_{e}$, after $\lambda$ training iterations. We will refer to this unit as the error unit. The distinction whether a new row or a new column is inserted is guided by the location of the most dissimilar neighboring unit to the error unit. Similarity is measured in the input space. 
Hence, we insert a new row or a new column depending on the position of the neighbor with the most dissimilar weight vector. The initialization of the weight vectors of the new units is simply performed as the average of the weight vectors of the existing neighbors. After the insertion the learning rate parameter $\alpha$ and the neighborhood function $h_{c i}$ are reset to their initial values and training continues according to the standard training process of SOMs. Note that we currently use the same value of the parameter $\tau_{m}$ for each map in each layer of the GHSOM.

Consider Fig. 1 for a graphical representation of the insertion of units. In this figure the architecture of the SOM prior to the insertion is shown on the left hand side where we find a map of $2 \times 3$ units with the error unit labeled by $e$ and its dissimilar neighbor signified by $d$. Since the most dissimilar neighbor belongs to another row within the grid, a new row is inserted between units $e$ and $d$. The resulting architecture is shown on the right hand side of the figure as a map of now $3 \times 3$ units.
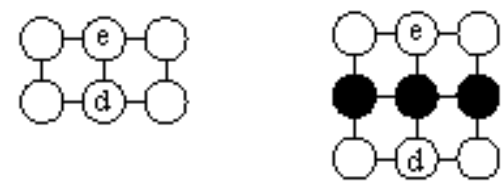

Fig. 1. Insertion of units

As soon as the growth process of the first layer map is finished, i.e. $\mathbf{M Q E} \mathbf{E}_{m}<\tau_{m} \mathbf{m q e}_{0}$, the units of this map are examined for expansion on the second layer. In particular, those units that have a large mean quantization error will add a new SOM to the second layer of the GHSOM. The selection of these units is based on the mean quantization error of layer 0 . A parameter $\tau_{u}$ is used to describe the desired level of granularity in input data discrimination in the final maps. More precisely, each unit $i$ fulfilling the criterion given in expression (4) will be subject to hierarchical expansion.

$$
\mathbf{m q e}_{i}>\tau_{u} \mathbf{m q e}_{0}
$$

The training process and unit insertion procedure now continues with these newly established SOMs. The major difference to the training process of the second layer map is that now only that fraction of input data is selected for training which is represented by the corresponding first layer map unit. The strategy for row or column insertion as well as the termination criterion is essentially the same as used for the first layer map. The same procedure is applied to any subsequent layers of the GHSOM.

The training process of the GHSOM is terminated when no more units require further expansion. Note that this training process does not necessarily lead to a balanced hierarchy, i.e. a hierarchy with equal depth in each branch. The depth of the hierarchy will rather reflect the un-uniformity, which should be expected in real world data collections.

Consider Fig. 2 for a graphical representation of a trained GHSOM. In particular, the neural network depicted in this figure consists of a single unit SOM at layer 0 , a SOM of $2 \times 3$ units in layer 1, six SOMs in layer 2, i.e. one for each unit in layer 1map. Note that each of these maps might have a different number and different arrangement of units as shown in the figure. Finally, there's one SOM in layer 3, which was expanded from one of the layer 2 units.

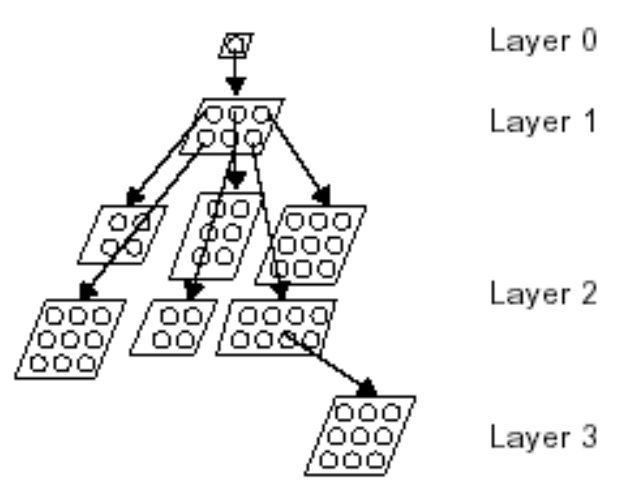

Fig. 2. Architecture of a GHSOM

To summarize, the growth process of the GHSOM is guided by two parameters $\tau_{u}$ and $\tau_{m}$. The parameter $\tau_{u}$ specifies the desired quality of input data representation at the end of the training process. Each unit $i$ with $\mathbf{m q e}_{i}>\tau_{u} \mathbf{m q e}_{0}$ will be expanded, i.e. a map is added to the next layer of the hierarchy, in order to explain the input data in more detail. Contrary to that, the parameter $\tau_{m}$ specifies the desired level of detail that is to be shown in a particular SOM. In other words, new units are added to a SOM until the MQE of the map is a certain fraction, $\tau_{m}$, of the mqe of its preceding unit. Hence, the smaller $\tau_{m}$ the larger will be the emerging maps. Conversely, the larger $\tau_{m}$ the deeper will be the hierarchy.

\section{Data Set}

For the experiments presented thereafter we use a collection of abstracts from the first International Conference on Intelligent Computing and Information Systems, ICICIS 2002. 
(http://asunet.shams.edu.eg/confs/icicis2002.html) as a sample document archive. ICICIS contains papers covering the areas of; fuzzy sets, rough sets, genetic algorithms, neural nets, data mining and knowledge discovery, expert systems, information storage and retrieval, web-based learning, medical informatics and others.

The documents can be thought of as forming topical clusters in the high-dimensional feature space spanned by the words that the documents are made up of. The goal is to map and identify those clusters on the 2-dimensional map display. Thus we use fulltext indexing to represent the various documents. In total, ICICIS consists of 68 papers containing 5417 content terms, i.e. terms used for document representation.

\subsection{Document Preprocessing}

For the training of SOMs, the documents must be encoded in form of numerical vectors. To be suited for the learning process of the map, to similar documents similar vectors have to be assigned. After training of the map, documents with similar contents should be close to each other, and possibly assigned to the same neuron. The presented approach is based on statistical evaluations of word occurrences. We do not use any information on the meaning of the words since in domains like scientific research we are confronted with a wide and (often rapidly) changing vocabulary, which is hard to catch in fixed structures like manually defined thesaurus or keyword lists. However, it is important to be able to calculate significant statistics. Therefore, the number of considered words must be kept reasonably small, and the occurrences of words sufficiently high. This can be done by either removing words or by grouping words with equal or similar meaning. A possible way to do so is to filter so-called stop words and to build the stems of the words. An overview of document pre-processing and encoding is given in Fig 3.
The idea of stop word filtering is to remove words that bear no content information, like articles, conjunctions, prepositions, etc. Furthermore, words that occur extremely often can be said to be of little information content to distinguish between documents. Also, words that occur very seldom are likely to be of no particular statistical relevance. Stemming tries to build the basic forms of words, i.e. strip the plural 's' from nouns, the 'ing' from verbs, or other affixes. A stem is a natural group of words with equal (or very similar) meaning. We currently used the stemming algorithm of [10], which uses a set of production rules to iteratively transform (English) words into their stems.

\subsection{Generating Characteristic Document Vectors}

Fig. 3 shows the principle of the proposed document encoding. At first, the original documents are preprocessed, i.e. they are split into words, then stop words are filtered and the word stems are generated. The occurrences of the word stems (frequencies) associated with the document are counted. A component in a $n$-dimensional vector is built, that characterizes the document. These vectors can be seen as the fingerprints of each document. For every document in the collection such a fingerprint is generated. Using GHSOM, these document vectors are then clustered and arranged into a 2-dimensional maps, the so-called document maps. Furthermore, each unit is labeled by specific keywords that describe the content of the assigned documents. The labeling method we used is based on methods proposed in [11]. It focuses on the distribution of words used in the documents assigned to the considered unit compared to the whole document database.

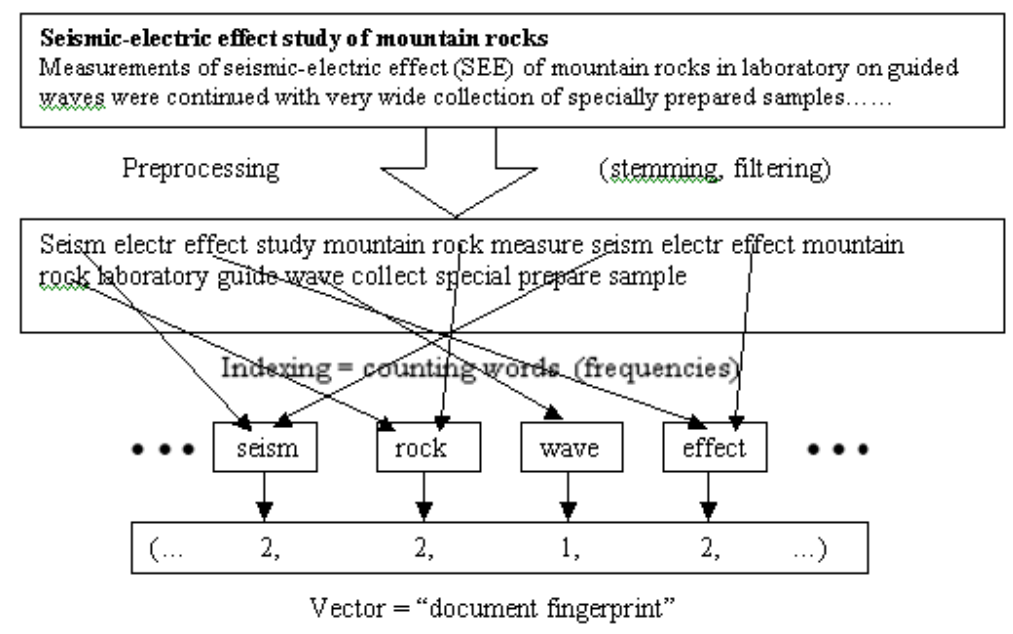

Fig. 3. Document preprocessing and encoding 


\section{Experimental Results and Discussion}

For our data set we trained both conventional selforganizing map and growing hierarchical SOM (GHSOM); to explain the difference and investigate the benefits of using GHSOM.

\subsection{Trained Conventional SOM}

Fig. 4 shows a conventional self-organizing map trained with the ICICIS abstracts data set. It consists of $5 \times 6$ units represented as table cells with a number of abstracts being mapped onto each individual unit (we refer to the abstract with symbol $T$ ). Each unit is labeled by specific keywords that describe the content of the assigned abstracts. The abstracts mapped onto the same or neighboring units are considered to be similar to each other in terms of the topic they deal with. We find, that the SOM has succeeded in creating a topology preserving representation of the topical clusters of abstracts. For example, in the lower left corner we find a group of units representing abstracts on the grid computing. To name just a few, we find abstracts T22, T23 on unit (5/1) ${ }^{1}$ covering resource scheduling in grid computing or T51, T53, T68 on unit (5/2) dealing with intrusion detection architecture for computational grids. A cluster of documents covering knowledge discovery and data mining is located in the upper left corner of the map around units (1/1) and (1/2), next to a cluster on genetic algorithms on units (1/3) and (2/3). Below this area, on units $(3 / 1),(3 / 2)$ and neighboring ones we find abstracts on neural networks. Similarly, all other units on the map can be identified to represent a topical cluster of news abstracts.

\subsection{Trained GHSOM}

Based on the artificial unit representing the means of all data points at layer 0 , the GHSOM training algorithm started with a $2 \times 2$ SOM at layer 1 . The training process for this map continued with additional units being added until the quantization error fell below a certain percentage of the overall quantization error of the unit at layer 0 . As mentioned earlier, the growth process of the GHSOM is guided by two parameters $\tau_{m}$ and $\tau_{u}$. We can say that, the smaller the parameter value $\tau_{m}$, the more shallow the hierarchy, and that, the lower the setting of parameter $\tau_{w}$, the larger the number of layers in the resulting GHSOM network will be.

\subsubsection{Deep Hierarchy}

Training the GHSOM with parameter $\tau_{m}=0.07$ and $\tau_{u}=0.0035$ results in a rather deep hierarchical structure of up to 4 layers. The layer 1 map is depicted in fig. 5(a) grows to a size of $4 \times 3$ units, all of which are expanded at subsequent layers. For convenience we list the topics of the various units, rather than the individual abstracts in the figure. For example, we find unit (2/1) to represent all abstracts related to knowledge discovery and data mining, whereas neural network topics are covered on unit $(2 / 2)$, or abstracts related to genetic algorithms on unit (4/1) in the lower left corner. Based on this first separation of the most dominant topical clusters in the abstract collection, further maps were automatically trained to represent the various topics in more detail. This results in 12 individual maps on layer 2, each representing the data of the respective higher-layer unit in more detail. Some of the units on these layer 2 maps were further expanded as distinct SOMs in layer 3.

We find the branch on data mining on unit $(2 / 1)$ of this map. This unit has been expanded to form a $2 \mathrm{x}$ 2 map in the second layer as shown in fig. 5(b). Unit $(1 / 1)$ of this map is dominated by abstracts related to enhancing algorithms for data mining, whereas, for example, abstracts focusing on mining medical data set are located in the lower left corner on unit (2/1). Other dominant cluster on this map is rough set. One unit of this second layer map is further expanded in a third layer. Unit (1/2) in the upper right corner representing abstracts related to data mining using statistical techniques. These abstracts are represented in more detail in the third layer.

\subsubsection{Shallow Hierarchy}

To show the effect of different parameter settings we trained a second GHSOM with $\tau_{m}$ set to half of the previous value $\left(\tau_{m}=0.035\right)$, while $\tau_{u}$, i.e. the absolute granularity of data representation, remained unchanged. This leads to a more shallow hierarchical structure of only up to 2 layers, with the layer 1 map growing to a size of $5 \times 4$ units depicted in fig. 6. Again, we find the most dominant branches to be, for example, genetic algorithms located on unit (1/3), data mining and knowledge discovery on unit (2/3), and neural networks on the lower right corner of this map. However, due to the large size of the resulting first layer map, a fine-grained representation of the data is already provided at this layer. This results in some larger clusters to be represented by two neighboring units already at the first layer, rather than being split up in a lower layer of the hierarchy. For example, we find the cluster on

\footnotetext{
${ }^{1}$ We use the notion $(x / y)$ to refer to the unit located in row $x$ and column $y$ of the map, starting with $(1 / 1)$ in the upper left corner
} 


\begin{tabular}{|c|c|c|}
\hline $\begin{array}{l}\text { use } \\
\text { result } \\
\text { introduce } \\
\text { Quality } \\
\text { algorithm }\end{array}$ & $\begin{array}{l}\text { inform } \\
\text { set } \\
\text { Model } \\
\text { E-business } \\
\text { accid }\end{array}$ & $\begin{array}{l}\text { paper } \\
\text { approach } \\
\text { method } \\
\text { design } \\
\text { structure }\end{array}$ \\
\hline down & down & down \\
\hline $\begin{array}{l}\text { paper } \\
\text { data } \\
\text { system } \\
\text { knowledge } \\
\text { Mine }\end{array}$ & $\begin{array}{l}\text { system } \\
\text { learn } \\
\text { network } \\
\text { neural } \\
\text { Artificial }\end{array}$ & $\begin{array}{l}\text { paper } \\
\text { use } \\
\text { high } \\
\text { apply } \\
\text { resource }\end{array}$ \\
\hline down & down & down \\
\hline $\begin{array}{l}\text { network } \\
\text { algorithm } \\
\text { recognize } \\
\text { image }\end{array}$ & $\begin{array}{l}\text { set } \\
\text { paper } \\
\text { education } \\
\text { system } \\
\text { web }\end{array}$ & $\begin{array}{l}\text { use } \\
\text { ability } \\
\text { database } \\
\text { base } \\
\text { data }\end{array}$ \\
\hline down & down & down \\
\hline $\begin{array}{l}\text { approach } \\
\text { genetic } \\
\text { propos } \\
\text { problem } \\
\text { algorithm }\end{array}$ & $\begin{array}{l}\text { paper } \\
\text { solve } \\
\text { problem } \\
\text { Graph } \\
\text { Optimum }\end{array}$ & $\begin{array}{l}\text { select } \\
\text { Draw } \\
\text { represent } \\
\text { algorithm } \\
\text { view }\end{array}$ \\
\hline down & down & down \\
\hline
\end{tabular}

(a) Layer 1 map: 4x3 units; Main topics

\begin{tabular}{|c|c|}
\hline $\begin{array}{l}\text { Mine } \\
\text { data } \\
\text { database } \\
\text { algorithm } \\
\text { Discovery }\end{array}$ & $\begin{array}{l}\text { data } \\
\text { algorithm } \\
\text { classify } \\
\text { system } \\
\text { statistic }\end{array}$ \\
\hline$\frac{\mathrm{T} 56}{\mathrm{~T} 57}$ & down \\
\hline $\begin{array}{l}\text { Mine } \\
\text { medical } \\
\text { data } \\
\text { knowledge } \\
\text { Information }\end{array}$ & $\begin{array}{l}\text { set } \\
\text { knowledge } \\
\text { algorithm } \\
\text { database } \\
\text { rough }\end{array}$ \\
\hline$\frac{\mathrm{T} 59}{\mathrm{~T} 61}$ & $\underline{\mathrm{T} 63}$ \\
\hline
\end{tabular}

(b) Layer 2 map: $2 \times 2$ units; Knowledge discovery

Fig. 5: Top and second level maps

\subsection{Comparison of Both Models (Conventional SOM and GHSOM)}

While we find the SOM to provide a good topologically ordered representation of the various topics found in the abstracts collection, no information about topical hierarchies can be identified from the resulting flat map. Apart from this we find the size of the map to be quite large with respect to the number of topics identified. This is mainly due to the fact that the size of the map has to be determined in advance, before any information about the number of topical clusters is available. GHSOM has two benefits over conventional selforganizing maps, which make this model particularly attractive in an information retrieval setting. First, GHSOM has substantially shorter training time than self-organizing map. The reason for that is, there is the obvious input vector dimension reduction on the transition from one layer to the next. Shorter input vectors lead directly to reduced training time because of faster winner selection and weight vector adaptation. Second, GHSOM may be used to produce disjoint clusters of the input data. Moreover, these disjoint clusters are gradually refined when moving down along the hierarchy. Contrary to that, the self-organizing map in its basic form cannot be used to produce disjoint clusters. The separation of data items is a rather tricky task that requires some insight into the structure of the input data. What one gets, however, from a self-organizing map is an overall representation of input data similarities. In this sense we may use the following picture to contrast the two 


\begin{tabular}{|c|c|c|c|}
\hline $\begin{array}{l}\text { time } \\
\text { algorithm } \\
\text { Optimum } \\
\text { tree } \\
\text { binary }\end{array}$ & $\begin{array}{l}\text { implement } \\
\text { algorithm } \\
\text { perform } \\
\text { Queue } \\
\text { Model }\end{array}$ & $\begin{array}{l}\text { algorithm } \\
\text { approach } \\
\text { problem } \\
\text { genetic }\end{array}$ & $\begin{array}{l}\text { approach } \\
\text { query } \\
\text { document } \\
\text { inform } \\
\text { retrieve }\end{array}$ \\
\hline$\underline{\text { down }}$ & $\underline{\mathrm{T} 12}$ & down & down \\
\hline $\begin{array}{l}\text { process } \\
\text { service } \\
\text { efficient } \\
\text { implement } \\
\text { paper }\end{array}$ & $\begin{array}{l}\text { paper } \\
\text { traffic } \\
\text { data } \\
\text { GPS } \\
\text { system }\end{array}$ & $\begin{array}{l}\text { Mine } \\
\text { data } \\
\text { algorithm } \\
\text { set } \\
\text { knowledge }\end{array}$ & $\begin{array}{l}\text { paper } \\
\text { compute } \\
\text { extract } \\
\text { result } \\
\text { match }\end{array}$ \\
\hline$\underline{\text { down }}$ & down & down & down \\
\hline $\begin{array}{l}\text { use } \\
\text { Model } \\
\text { technique } \\
\text { process } \\
\text { work }\end{array}$ & $\begin{array}{l}\text { control } \\
\text { design } \\
\text { estimate } \\
\text { system } \\
\text { aircraft }\end{array}$ & $\begin{array}{l}\text { system } \\
\text { resource } \\
\text { network } \\
\text { compute } \\
\text { apply }\end{array}$ & $\begin{array}{l}\text { result } \\
\text { paper } \\
\text { compare } \\
\text { algorithm }\end{array}$ \\
\hline down & down & down & down \\
\hline $\begin{array}{l}\text { Model } \\
\text { evolve } \\
\text { software } \\
\text { process }\end{array}$ & \begin{tabular}{|l|} 
variable \\
determine \\
classify \\
rule \\
system
\end{tabular} & $\begin{array}{l}\text { network } \\
\text { propos } \\
\text { grid } \\
\text { describe } \\
\text { study }\end{array}$ & $\begin{array}{l}\text { algorithm } \\
\text { interest } \\
\text { Arab } \\
\text { segment } \\
\text { Character }\end{array}$ \\
\hline down & down & down & down \\
\hline $\begin{array}{l}\text { system } \\
\text { balance } \\
\text { load } \\
\text { heterogeneous } \\
\text { distribute }\end{array}$ & $\begin{array}{l}\text { neural } \\
\text { set } \\
\text { Artificial } \\
\text { system } \\
\text { level }\end{array}$ & $\begin{array}{l}\text { paper } \\
\text { network } \\
\text { neural } \\
\text { operation } \\
\text { level }\end{array}$ & $\begin{array}{l}\text { learn } \\
\text { network } \\
\text { neural } \\
\text { regress } \\
\text { recognition }\end{array}$ \\
\hline down & $\mathrm{T} 24$ & down & down \\
\hline
\end{tabular}

Fig. 6: Layer 1 map: 5x4 units shallow hierarchy

models of neural networks. Self-organizing maps can be used to produce maps of the input data whereas GHSOM produces an atlas of the input data. Taking up this metaphor, the difference between both models is quite obvious. Selforganizing maps, in our point of view, provide the user with a single picture of the underlying data archive. As long as the map is not too large, this picture may be sufficient. As the maps grow larger, however, they have the tendency of providing too little orientation for the user. In such a case we would advise to change to GHSOM as the model for representing the contents of the data archive. In this case, the data is organized hierarchically, which facilitates browsing into relevant portions of the data archive.

\section{Conclusions and Future Work}

We presented the GHSOM, a novel neural network model based on the self- organizing map. The main feature of this model is its capability of dynamically adapting its architecture to the requirements of the input space. Instead of having to specify the precise 
number and arrangement of units in advance, the network determines the number of units required for representing the data at a certain accuracy level at training time. This growth process is guided solely by the desired granularity of data representation. As opposed to other growing network architectures, the GHSOM does not grow into a single large map, but rather dynamically evolves into a hierarchical structure of growing maps in order to represent the data at each level in the hierarchy at certain granularity. This enables the creation of smaller maps, resulting in better cluster separation due to the existence of separated maps. It further allows easier navigation and interpretation by providing a better overview of huge data sets.

We demonstrated that both the self-organizing map and the hierarchical feature map are highly useful for assisting the user to find his or her orientation within the document space. The shortcoming of the self-organizing map, however, is that each document is shown in one large map and thus, the borderline between clusters of related and clusters of unrelated documents are sometimes hard to find. This is especially the case if the user does not have sufficient insight into the contents of the document collection. The GHSOM overcomes this limitation in that the clusters of documents are clearly visible because of the architecture of the neural network. The document space is separated into independent maps along different layers in a hierarchy. The similarity between documents is shown in a finegrained level in maps of the lower layers of the hierarchy while the overall organizational principles of the document archive are shown at higher layer maps. Since such a hierarchical arrangement of documents is the common way of organizing conventional libraries, only small intellectual overhead is required from the user to find his or her way through the document space.

An important feature of GHSOM is that, the training time is largely reduced by training only the necessary number of units for a certain degree of detail representation. The benefits of the proposed approach have been demonstrated by a real world application from the text classification domain.

Our future work on GHSOM includes fine-tuning the basic algorithm and applying it to collections in any language, provided that words as primary tokens can be identified. This may require special preprocessing steps for languages as Chinese, where word boundaries are not eminent from the texts. In addition, develop a method for setting the threshold values $\left(\tau_{m}\right.$ and $\left.\tau_{u}\right)$ automatically according to application requirements.
References:

[1] T. Kohonen, "Self-organized formation of topologically correct feature maps," Biol. Cybern. vol. 43, 1982, pp. 59-69.

[2] T.Kohonen, "Self-organizing maps" Berlin, Germany: Springer verlage, 1998.

[3] K. Lagus, T. Honkela, S. Kaski, and T. Kohonen, "Self-organizing maps of document collection:

A new approach to interactive exploration" In Proc. Int. Conf. on Knowledge Discovery and Data Mining (KDD-96), Portland, OR, vol.36, 1998, pp. 314-322

[4] D. Merkl, "Exploration of text collections with hierarchical feature maps". In Proc. Int. ACM

SIGIR Conf. on Information Retrieval (SIGIR'97), Philadelphia, PA, vol.62, 1997,pp. 412-419

[5] A. Rauber and D. Merkl, "Finding structure in text archives" In Proc. Europe an Symp. on Artificial Neural Networks (ESANN98), Bruges, Belgium, vol.18, 2000,pp.410-419

[6] B. Fritzke, "Growing self-organizing networks -Why?" In Proc. Europ Symp on Artificial

Neural Networks (ESANN'96), Bruges, Belgium, vol.16,1998,pp.222-230.

[7] B. Fritzke, "Growing grid: a self-organizing network with constant neighborhood range and adaptation strength" Neural Processing Letters, 1997.

[8] R. Miikkulainen, "Script recognition with hierarchical feature maps" Connection Science, 2, 1995.

[9] M. Salem, M. Syiam, and A. F. Ayad, "Improving self-organizing feature map (SOFM) training algorithm using k-means initialization" In Proc. Int. Conf. on Intelligent Eng. Systems INES, IEEE, vol.40,2003,pp.41-46.

[10] M. Porter, "An algorithm for suffix stripping" Program 14(3), pp. 130-137, 1980.

[11] K. Lagus, and S. Kaski, "Keyword selection method for characterizing text document maps"

In Proc of ICANN99, Ninth International Conference on Artificial Neural Networks,IEEE,vol 68, 1999,pp.615-623. 